\title{
Formation of a mixed ordered termination on the surface of $\mathrm{LaAlO}_{3}(001)$
}

\author{
A. J. H. van der Torren, S. J. van der Molen, and J. Aarts \\ Huygens - Kamerlingh Onnes Laboratorium, Leiden University, Niels Bohrweg 2, 2300 RA Leiden, The Netherlands
}

(Received 8 February 2015; revised manuscript received 26 May 2015; published 22 June 2015)

\begin{abstract}
We have investigated the surface termination of $\mathrm{LaAlO}_{3}(001)$ at elevated temperatures by low-energy electron microscopy. The terminating layer can be $\mathrm{LaO}$ or $\mathrm{AlO}_{2}$. The $\mathrm{LaO}$ surface shows a $\sqrt{5} \times \sqrt{5} \mathrm{R} 26$ reconstruction which can be used as a signature for the $\mathrm{LaO}$ termination, while the $\mathrm{AlO}_{2}$ termination is unreconstructed. We find that heating of as-delivered substrates in vacuum or heating substrates which were previously annealed in air or oxygen can lead to a reconstructed surface as observed in diffraction. However, the real-space image shows that the reconstructed areas only cover about one-third of the surface and that the termination is actually an ordered mixture of the reconstructed $\mathrm{LaO}$ and the unreconstructed $\mathrm{AlO}_{2}$ terminations. This conclusion is supported by data from atomic force microscopy. We also demonstrate how the disordered mixture of both terminations changes to large $\mathrm{LaO}$ islands in the middle of the $\mathrm{AlO}_{2}$ terraces upon heating.
\end{abstract}

DOI: 10.1103/PhysRevB.91.245426

PACS number(s): 68.35.B-, 68.37.Nq, 68.37.Ps

\section{INTRODUCTION}

Much effort is currently directed to investigating the properties of interfaces between perovskite oxides, in particular those of the $\mathrm{ABO}_{3}$ type, with particular emphasis on the twodimensional electron gas (2-DEG) which is formed between the insulators $\mathrm{SrTiO}_{3}$ (STO) and $\mathrm{LaAlO}_{3}(\mathrm{LAO})$ [1]. Of prime importance here is control over and knowledge of the atomic termination layer of the surface on which the interface is prepared. For instance, in order to form the 2-DEG, it is well known that the STO surface has to be terminated with a $\mathrm{TiO}_{2}$ layer. Recipes exist for the fabrication of different termination layers on the various substrates which are used in oxide research. Of recent interest are endeavors to fabricate mixed ordered terminations, which allow for the possibility to perform selective area growth.

In this study, we focus on the surface of $\mathrm{LaAlO}_{3}(001)$, which can be $A$-site terminated with a layer of $\mathrm{LaO}$ or $B$-site terminated with a layer of $\mathrm{AlO}_{2}$. In the literature, contradictory reports can be found on the issue of fabricating singly terminated surfaces. Kim et al. [2] use an $1100^{\circ} \mathrm{C}$ annealing step in oxygen and find a predominantly $\mathrm{AlO}_{2}$ termination, while Gunnarsson et al. [3] come to the conclusion that the surface is doubly terminated when only annealed in oxygen. They report that etching the surface with $\mathrm{HCl}$, followed by annealing in oxygen at the relatively low temperature of $800^{\circ} \mathrm{C}$ leads to $\mathrm{AlO}_{2}$ termination [3]. Early work using ion scattering experiments [4] and photoemission [5] suggested that starting from a $B$-site termination, a complete $A$-site termination can also be obtained even at $250^{\circ} \mathrm{C}$, but careful measurements of the temperature dependence of the surface structure using synchrotron x-ray radiation found no evidence for such a conversion. Rather, it was shown that the structural relaxation which occurs in the top layers at elevated temperatures is enough to mimic an $A$-site termination in scattering experiments [6]. This may also explain why an $\mathrm{LaO}$-terminated surface was reported after annealing at $700^{\circ} \mathrm{C}$ in UHV using $\mathrm{He}$ atom scattering [7], while similar annealing was found to yield an $\mathrm{AlO}_{2}$ surface with $10 \mathrm{~nm} \mathrm{LaO}$ islands in experiments involving scanning tunneling microscopy [8].

Extensive surface $x$-ray measurements were performed by Lanier et al. on surfaces which were prepared by annealing an
$\mathrm{LaAlO}_{3}$ substrate between $1100^{\circ} \mathrm{C}$ and $1500^{\circ} \mathrm{C}$ in air [9]. Such surfaces show a clear $\sqrt{5} \times \sqrt{5}$ R26 (RT5) reconstruction, and it was concluded that it is the $\mathrm{LaO}$ layer which reconstructs, yielding a surface with one La vacancy per surface unit cell and stoichiometry ( $\left.V \mathrm{La}_{4} \mathrm{O}_{5}\right)$ (with $V$ the vacancy). In this study, the question of the amount of $\mathrm{LaO}$ coverage was not addressed. We are not aware of a study of the occurrence of reconstructions on the $\mathrm{AlO}_{2}$-terminated surface, and as a working hypothesis we assume that it does not reconstruct.

Here we use low-energy electron microscopy (LEEM) to study the behavior of the $\mathrm{LaAlO}_{3}$ surface at high temperatures, an issue which is both relevant for surface preparation and for growth. With the LEEM technique, we are able to discern different terminations in two ways. One is through the diffraction pattern when different reconstructions occur on the two terminations. By using apertures in the outgoing beams, LEEM also allows us to make a real-space image of the occurrence of different reconstructions with high lateral resolution. The other way is to measure the intensity of the diffraction spots $(I)$, in particular of the specular beam, as a function of the electron energy $(V)$.

In this way, we find for $\mathrm{LaAlO}_{3}[001]$ that surfaces can be prepared with a single termination of $\mathrm{AlO}_{2}$, but that high-temperature treatments, presumably yielding a reconstructed $\mathrm{LaO}$ surface, actually lead to spatially separated areas with and without reconstruction and with different $I V$ characteristics. Complementing the LEEM data with roomtemperature atomic force microscopy (AFM), we conclude that the annealing process leads to a mixed but ordered termination of both $\mathrm{LaO}$ and $\mathrm{AlO}_{2}$, with the $\mathrm{LaO}$ termination occurring predominantly in the middle of terraces on the otherwise atomically flat surface.

\section{EXPERIMENT}

The LEEM experiments were performed in the so-called Escher setup, a state-of-the-art aberration-corrected LEEM facility at Leiden University [10-13]. In the LEEM, electrons are accelerated from a cold-field emission gun to an energy of $15 \mathrm{kV}$ and deflected by a magnetic prism array over $90^{\circ}$ towards the objective lens and the sample. Between the lens 
and the sample, a decelerating field is applied, which is used to accurately tune the landing energy of the electrons on the sample in the range from 0 to $100 \mathrm{eV}$. The zero landing energy is influenced by the difference between sample and gun voltage supply, the work function of the gun filament and the work function of the sample. Determining the correct value for the zero of energy can, in principle, be done with an inaccuracy of less than $0.1 \mathrm{eV}$, but in this work we did not pay special attention to the optimization, leading to sample-to-sample variations of the order of $1 \mathrm{eV}$. At the sample, the electrons are diffracted and accelerated again towards the objective lens. After passing the objective lens, they are deflected into the projection column and pass through a second magnetic prism deflecting the beam to an electrostatic mirror, a procedure which corrects for chromatic aberration [10]. Coming back from the mirror, the electrons reenter the projection column and form a real-space or reciprocal-space image on the channel plate detector.

With this system, various imaging modes can be used. In the first place, low-energy electron diffraction (LEED) can be used to study the structure of the surface. Lateral variations in the structure can be imaged by placing an aperture in the diffraction plane around one of the diffraction spots. By changing the projector lenses to produce a real-space image, we can then study which areas of the sample contribute to that diffraction condition. This is called dark-field imaging. For the so-called bright-field mode, the aperture is placed around the zeroth-order LEED spot. Here we can still have contrast between structures with different reflection intensities (given by the structure factor) at a given electron energy. By measuring the intensity of the diffracted beam as a function of electron energy at the sample, i.e., the so-called $I V$ curve, a specific signature of the surface can be obtained [14-16]. Since the $I V$ curve of a surface is determined by the precise positions of the atoms, contrast between reconstructed and nonreconstructed areas can also be found at certain energies in the bright-field mode. To determine which areas in the bright field belong to which reconstruction, the dark-field images are used.

Experiments in the LEEM are performed in a vacuum of $10^{-9}$ mbar, and the sample can be continuously heated up to $1300{ }^{\circ} \mathrm{C}$. Images are acquired with an acquisition time of $250 \mathrm{~ms}$ (four images per second) by a $1280 \times 1024$ pixel CCD camera behind a channel plate detector. Switching within less than a second between different magnifications and image modes results in fast real-time imaging of changes in the surface morphology.

Atomic force microscopy was routinely used to characterize the surface topography. A special experiment was performed on two samples to compare the information of LEEM and AFM on the same area. For this, trenches were etched in the surface using electron beam lithography and Ar-ion etching. These trenches served as markers both in the light microscope attached to the AFM and in the photoemission mode of the LEEM, both of which have a field of view of a few hundred micrometers.

Commercial substrates, polished on one side, were obtained from crystal $\mathrm{GmbH}$ in three different batches. For experiments where samples were annealed in air, the substrates were first loaded into a furnace and heated up to $1150^{\circ} \mathrm{C}$. After annealing, they were loaded into the LEEM. Other samples were directly loaded into the LEEM and annealed in vacuum. The temperature in the LEEM was measured by a pyrometer assuming an emissivity of 0.9 . During imaging, samples were kept at at least $400{ }^{\circ} \mathrm{C}$ to avoid charging of the insulating substrates.

\section{RESULTS}

An issue which surfaced in the research described here, but which may well be more general, was that the as-received samples did not all behave in exactly the same manner. In the first set of experiments, a diffraction pattern pointing to a surface reconstruction could be directly obtained by annealing in vacuum at high temperatures, but several attempts to make an $\mathrm{AlO}_{2}$-terminated surface by annealing in oxygen failed. With a different batch of substrates, this $(B$-site) termination could be prepared, but obtaining the $(A$-site) reconstructed surface is only possible for a part of the surface. This will be detailed below.

\section{A. Reconstructed surfaces}

In Fig. 1(a), we show the diffraction pattern of an asreceived sample, taken at $875^{\circ} \mathrm{C}$, which shows an unreconstructed surface. The sample was then annealed in situ for about seven hours at a temperature of $1100{ }^{\circ} \mathrm{C}$ and cooled to $910^{\circ} \mathrm{C}$. This resulted in the diffraction pattern given in Fig. 1(b). It has four extra spots deriving from a rotated square surface net indicative of the RT5 reconstruction. Moreover, as indicated in the figure, the extra spots are doubled due to the fact that there are two equivalent rotations of the reconstruction, and both types are formed as domains on the surface. Figure 2 shows the $I V$ curve of the $(0,0)$ spot for the surface before and after annealing. They are quite similar, although the curve after annealing shows a more pronounced maximum at $18 \mathrm{~V}$ and an additional peak at $22 \mathrm{~V}$.

With the two equivalent fractional order patterns in the LEED pattern, the dark-field mode can be used to image the spatial extent of the two reconstructed domains separately. This is shown in Fig. 3(a), where the two RT5 domains are color
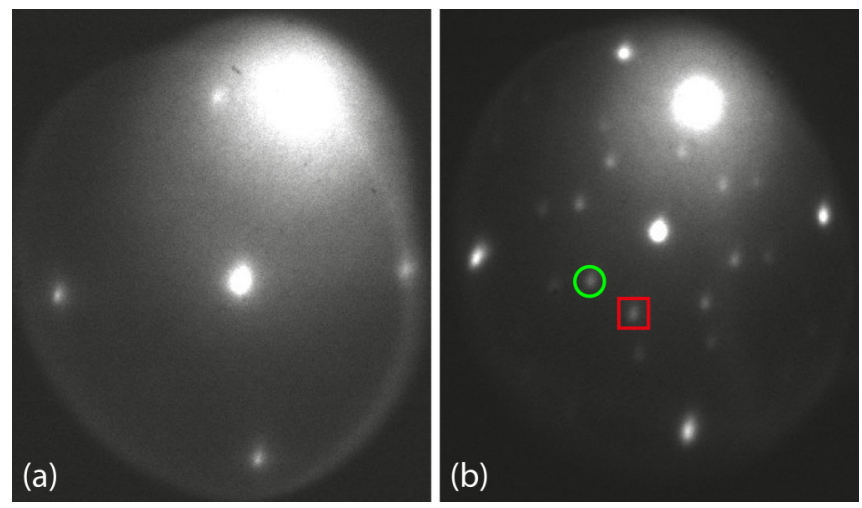

FIG. 1. (Color online) LEED pattern (a) before and (b) after annealing the sample at $1100^{\circ} \mathrm{C}$. The images were taken at $17 \mathrm{eV}$ and at $875^{\circ} \mathrm{C}$ and $910^{\circ} \mathrm{C}$, respectively. In (b), the diffraction spots of the $\sqrt{5} \times \sqrt{5}$ R26 reconstruction are visible. Spots from two distinct domains are denoted with a green circle and a red square. The bright spot in the top right corner is due to secondary scattered electrons which are displaced from the center by dispersion in the system. 


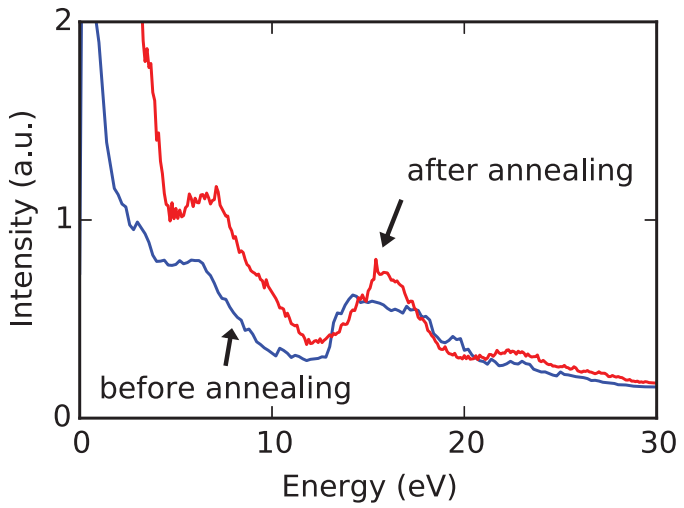

FIG. 2. (Color online) LEED- $I V$ curves taken from the $(0,0)$ spots of the LEED patterns shown in Fig. 1, before and after annealing the sample.

coded in red and green. Interestingly, the reconstruction does not fully cover the surface. Rather it forms in stripelike patterns with a more or less random variation of the two rotations, covering no more than about one-third of the surface. The larger fraction of the surface is therefore not reconstructed. The reconstruction can also be imaged in bright field by choosing the appropriate energy, as shown in Fig. 3(b) taken at $17.6 \mathrm{eV}$. The two types of rotation are now not distinguishable, but otherwise the image faithfully reproduces the areas where the reconstruction is present (bright) or absent (dark). By taking bright-field images at different electron energies, it is also possible to generate $I V$ curves at all positions on the surface. Figure 4 shows such $I V$ curves of the $(0,0)$ beam, taken on either a reconstructed (bright) or unreconstructed (dark) area. They are quite different. The unreconstructed area shows peaks around $5,9,12$, and $18 \mathrm{~V}$, plus a small maximum around $25 \mathrm{~V}$. The one from a reconstructed area shows peaks around 10 and $18 \mathrm{~V}$ and generally shows a higher intensity, which leads to the contrast seen in Fig. 3(b) taken at 17.6 V. Comparing these data to the $I V$ curves in Fig. 2 emphasizes that $I V$ curves taken on surfaces with different structures are of limited value. In particular, the curve after annealing represents some weighted average of the two curves in Fig. 3, but cannot be used as the characteristic of either one of the surface terminations.

Although difficult to see, in Fig. 3(b) there are also thin black lines visible in the bright-field image, which are related

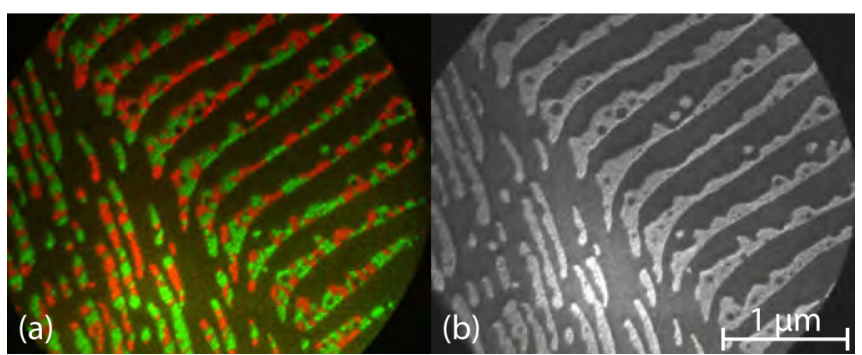

FIG. 3. (Color online) (a) Multi-dark-field image and (b) brightfield image of $\mathrm{LaAlO}_{3}$ after annealing above $1070^{\circ} \mathrm{C}$. The images were taken at 15 and $17.6 \mathrm{eV}$, respectively, at a temperature of $890^{\circ} \mathrm{C}$. The dark-field image consists of the two images of the distinct RT5 domains, overlaid in green and red.

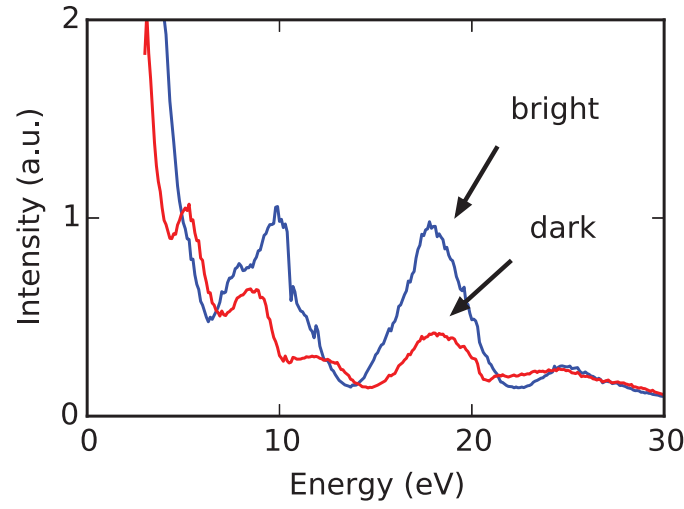

FIG. 4. (Color online) IV curves taken from a selected area of the zero-order beam and taken from the reconstructed (bright) and unreconstructed (dark) areas on the surface. A circle containing 1264 pixels (equivalent to a diameter of about $100 \mathrm{~nm}$ ) within a brightfield image is averaged for every energy. Figure 3(b) is one of the bright-field images out of this energy series.

to and localized at step edges on the substrate. They can be seen to bend over roughly $90^{\circ}$ in the field of view, with the stripelike domains following the direction of the steps. The relation between steps and domains was investigated further. For this, we used a sample with Ar-ion etched markers as described in the previous section. Figure 5(a) shows a bright-field image taken at $15.2 \mathrm{eV}$, an energy at which the step contrast is enhanced. Two steps are visible, indicated by red arrows, and it is seen that the reconstructed area lies in the middle of the terrace, which is defined by the two steps. Figure 5(b) shows an AFM image made on the same area. The steps are sharp and clearly visible, but also the reconstructed area is visible in the AFM image, which therefore appears to be of different height. The cross section from the AFM image, given in Fig. 5(d), shows that the steps around the reconstruction are about $0.35 \mathrm{~nm}$ high, corresponding to a full $\mathrm{LaAlO}_{3}$ unit cell. The steps bordering the reconstruction are smaller, only about $0.1 \mathrm{~nm}$, and the reconstructed areas lie deeper than the terrace on which they reside. The smaller steps can also be observed in the bright-field
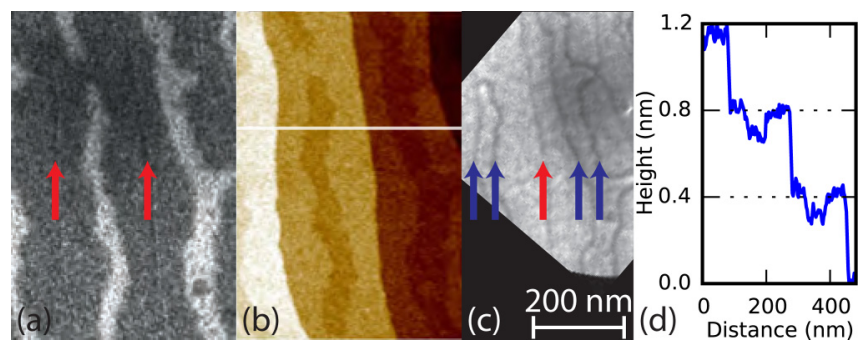

FIG. 5. (Color online) (a) Bright-field image of $\mathrm{LaAlO}_{3}$ at $15.2 \mathrm{eV}$. Dark lines, indicated by red arrows, are unit-cell steps between substrate terraces. The bright area is the $\sqrt{5} \times \sqrt{5} \mathrm{R} 26$ reconstructed area. (b) AFM image of the same area. The thin white bar indicates the position of the cross section shown in (d). This cross section is averaged over seven lines in the image. (c) Bright-field image taken at $26.8 \mathrm{eV}$. The red arrow again indicates the step between the terraces, while the blue arrows indicate the step between the terrace and the reconstruction. (d) Height profile of the area given by the white line in (b). 


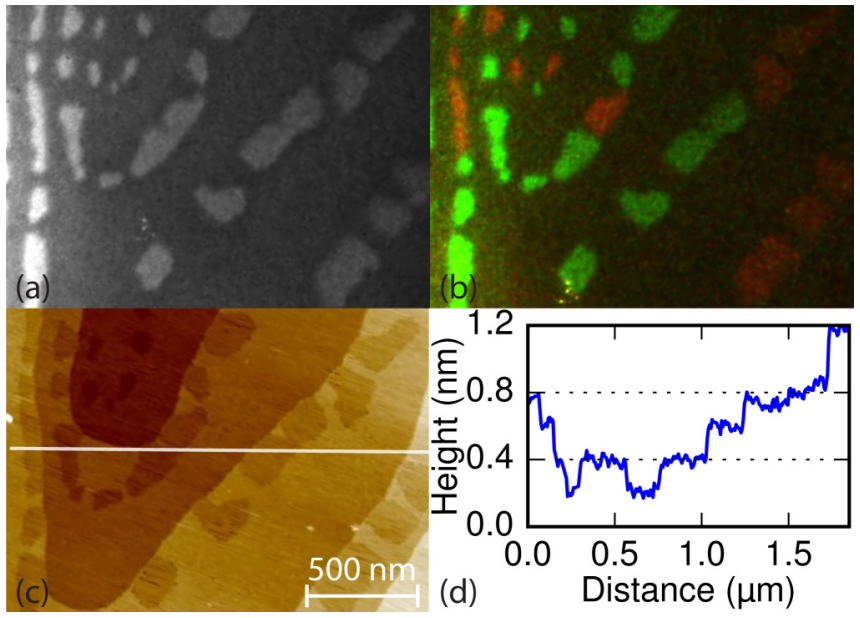

FIG. 6. (Color online) (a) Bright-field image at $9.2 \mathrm{eV}$ and (b) dark-field images at $14 \mathrm{eV}$ of an $\mathrm{LaAlO}_{3}$ sample annealed in air; (c) AFM image of the same area. According to the AFM image, the reconstructed areas clearly lie lower than the surrounding terraces. (d) Height profile along the cross section drawn in (c). Both half unit-cell steps and full unit-cell steps are present. The half unit-cell steps correspond to the reconstructed areas, while the full unit-cell steps are between two unreconstructed terraces.

image at the appropriate energy. Figure 5(c) shows an image taken at $26.8 \mathrm{eV}$. This energy is chosen such that there is no contrast from the reconstruction, but there is (phase) contrast to observe the steps. The middle red arrow shows the right-hand step in the earlier image, but four extra steps have appeared (outer blue arrows) which border the reconstructed areas.

Most experiments in the literature reporting the occurrence of the RT5 reconstruction and/or a mixed termination were performed by extensive annealing of the $\mathrm{LaAlO}_{3}$ sample in air or oxygen, rather than in UHV as described above. We therefore performed annealing experiments in air on samples from the third batch. One sample was annealed for ten hours in a tube furnace at $1150^{\circ} \mathrm{C}$. Figure 6(a) shows the resulting LEEM and AFM images, which are very comparable to the UHV annealed samples. The atomically flat patches in the AFM image are now somewhat larger, which makes the determination of the height variations easier. As before, it is seen that the reconstructed area lies deeper than the surrounding terrace, but the stripes now tend to lie closer to the steps rather than in the middle of the terraces. The steps between the reconstructed and unreconstructed areas are clearly of half unit-cell height. This was less clear in Fig. 5, which could be caused by incomplete $\mathrm{LaO}$ termination. Looking closely at Fig. 5(a) again reveals numerous small dark spots within the bright area. Possibly the $\mathrm{LaO}$ termination is not fully developed and small $\mathrm{AlO}_{2}$ islands still remain. Averaging such $\mathrm{AlO}_{2}$ areas with the $\mathrm{LaO}$ areas might result in an AFM height profile with less than half unit-cell steps.

\section{B. The singly terminated surface}

To further understand the difference between the reconstructed and unreconstructed areas, we prepared a sample with a single terminated surface. As described in Sec. I, how to prepare a single $\mathrm{AlO}_{2}$ termination is not completely obvious.
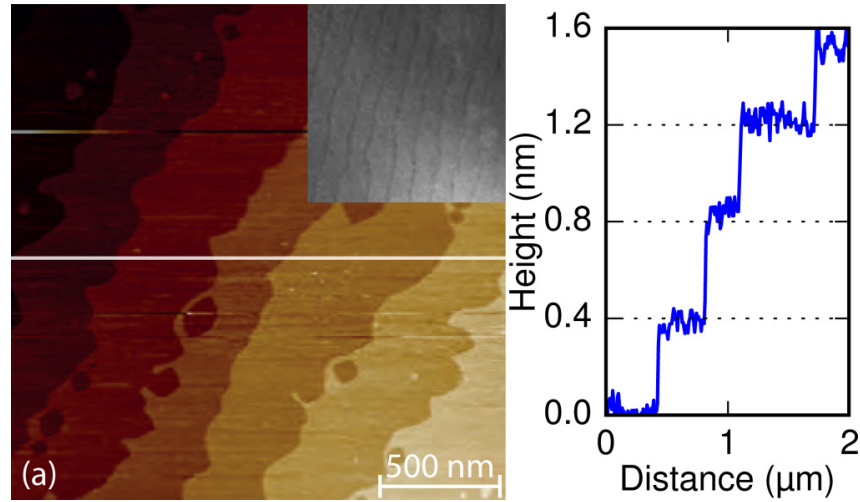

FIG. 7. (Color online) (a) AFM image of a singly $\mathrm{AlO}_{2}$ terminated sample; (b) height profile taken along the white line drawn in (a). Only full unit-cell steps can be seen, confirming that the sample is singly terminated. Inset: A bright-field image of this surface, taken at $850^{\circ} \mathrm{C}$ and $15.2 \mathrm{eV}$. The width of the image is $1 \mu \mathrm{m}$.

Samples from two batches were annealed in various ways, i.e., in oxygen at atmospheric pressure as well as in air and vacuum. Some were etched in $\mathrm{HCl}$, as reported in Ref. [3], while others were directly annealed, as reported by Ref. [17]. None of them led to the desired unit-cell-step and terrace structure as reported, e.g., in Refs. [3,17]. In a third batch, we succeeded using a two-step process. The sample was first annealed at $800^{\circ} \mathrm{C}$ in $\mathrm{UHV}$, followed by exposure to air after cooling down to room temperature. A subsequent anneal at $1000^{\circ} \mathrm{C}$ in $\mathrm{UHV}$ resulted in an unreconstructed surface. An AFM image of this surface is presented in Fig. 7(a). Single steps with a height of $0.4 \mathrm{~nm}$ are visible [see Fig. 7(b)], and equally important, no half-size steps were found as in Fig. 5(d). A bright-field image, taken at $850^{\circ} \mathrm{C}$ and $15.2 \mathrm{eV}$, showed terraces of the same size as in AFM (see inset, Fig. 7). All data indicate that the surface is singly terminated, and presumably by $\mathrm{AlO}_{2}$. A LEED- $I V$ curve taken on this surface is shown in Fig. 8. Comparison of this $I V$ curve with the one given in Fig. 4 of a dark (unreconstructed) area on the reconstructed sample shows them to be virtually identical. From this, we conclude that the

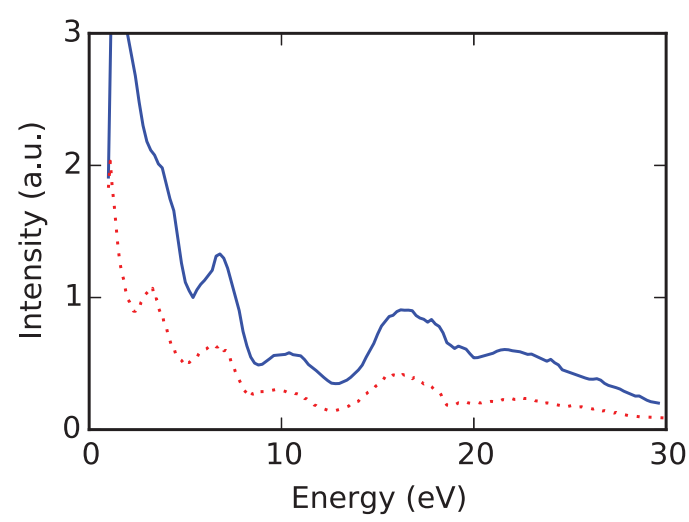

FIG. 8. (Color online) LEED- $I V$ curve of the single terminated sample shown in Fig. 7 (blue line). The $I V$ curve of the unreconstructed area shown in Fig. 4 is shown for comparison (red dots). This curve was shifted by $2 \mathrm{eV}$ since the zero of energy was not determined accurately. 


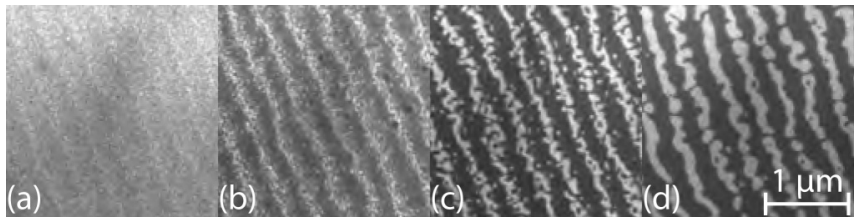

FIG. 9. Bright-field images of $\mathrm{LaAlO}_{3}$ surface. The images are taken during annealing at $935,1090,1155$, and $1170{ }^{\circ} \mathrm{C}$, respectively. The landing energy of the electrons is around $16.5 \mathrm{eV}$.

unreconstructed part of such a singly terminated surface is also $\mathrm{AlO}_{2}$ terminated.

\section{On the formation of the mixed ordered termination}

Being able to prepare and image the partial RT5 reconstruction and $\mathrm{LaO}$ termination by $\mathrm{UHV}$ annealing as discussed in Sec. III A offers a unique opportunity to follow the process which leads to the mixed ordered termination. The first signs of the RT5 LEED pattern appear at a temperature of $930^{\circ} \mathrm{C}$, the lowest temperature where we can probe the mixed ordered termination. Figure 9 shows four bright-field images during the annealing process, taken at $935,1090,1155$, and $1170{ }^{\circ} \mathrm{C}$ at an energy around $16.5 \mathrm{~V}$. The first image shows a grainy structure which starts to form small stripes and spots in the second image. When increasing the temperature, the small spots form larger continuous areas. In the last image, the small dots have mostly disappeared and the bright areas form wide and continuous stripes. Looking back at the first image, it suggests that there the reconstructed areas are very small, leading to relatively little coherent scattering. By repeating the experiment with different time scales, we find no real differences. Going to $1200^{\circ} \mathrm{C}$ in a few seconds or in an hour does not change the result. Also keeping the sample close to $1200^{\circ} \mathrm{C}$ for seven hours did not change the morphology which formed initially.

\section{DISCUSSION}

Taking the data together, the first point to make is that we found variety in the way that samples from different batches behaved. Nevertheless, several clear conclusions can be drawn. A singly terminated $\mathrm{AlO}_{2}$ surface can be prepared, although the procedure may vary. Without being complete, in the literature, a high-temperature $\left(1000^{\circ} \mathrm{C}\right)$ anneal in flowing oxygen was used [2], a somewhat lower $\left(800^{\circ} \mathrm{C}\right)$ flowing oxygen anneal preceded by a cleaning step in $\mathrm{HCl}$ was used [3], or a $900{ }^{\circ} \mathrm{C}$ anneal at very low oxygen pressure $\left(10^{-6} \mathrm{mbar}\right)$ was used [17]. This already shows that the recipe is not unique. We did not find single termination by these methods, but succeeded through a two-step process, i.e., an $800^{\circ} \mathrm{C}$ anneal in UHV, followed by exposure to air and a $1000{ }^{\circ} \mathrm{C}$ anneal in UHV. This suggests that the process for preparing single termination depends on the history of the sample, possibly even including the polishing process. A successful procedure leads, in our case, to an atomically flat surface with only unit-cell steps of $0.4 \mathrm{~nm}$ height. This $\mathrm{AlO}_{2}$-terminated surface is unreconstructed and has a well-defined and unique LEED- $I V$ signature.

Using the occurrence of the RT5 reconstruction as a signature for $\mathrm{LaO}$ termination, we come to the conclusion that a singly terminated $\mathrm{LaO}$ surface cannot be prepared. Preparing a reconstructed surface either by annealing in UHV or in air, we find from imaging by LEEM (both in bright field and in dark field) that the reconstructed areas form in stripelike domains. From the step contrast, it is seen that the stripes tend to be found in the middle of terraces, and the reconstructed $\mathrm{LaO}$ stripes have a LEED- $I V$ signature which is different from the $\mathrm{AlO}_{2}$ surface. Moreover, the unreconstructed part of the terraces do show the $\mathrm{AlO}_{2}$ signature. From the AFM data, we find that the reconstructed areas lie lower than the surrounding terraces, with a height difference of about half a unit cell. Our results indicate that the reconstruction starts forming at $930^{\circ} \mathrm{C}$ when the RT5 LEED spots become visible and small islands of reconstructed surface are seen to form. This is roughly consistent with the results of Schmidt et al. [8], who find a mixture of small $\mathrm{LaO}$ islands and $\mathrm{AlO}_{2}$ islands spread over the surface after annealing at $670^{\circ} \mathrm{C}$. The presence of small islands might also be the explanation of the presence of steps less than half a unit cell in Fig. 5. In Fig. 5(a), the bright area still has small black speckles, indicating the $\mathrm{LaO}$ islands are not fully grown together and small $\mathrm{AlO}_{2}$ islands are present within the $\mathrm{LaO}$ area. These differences are not resolved by the AFM and are averaged in the picture, resulting in the small steps. Finally, we note the fact that the LaO-based areas lie deeper than the $\mathrm{AlO}_{2}$ surface. This suggests a scenario in which the starting surface has a mixed termination with $\mathrm{AlO}_{2}$ islands on top. Increasing the temperature mobilizes the islands which seek step edges to form closed $\mathrm{AlO}_{2}$ layers not fully covering the surface. At the same time, the lower-lying exposed $\mathrm{LaO}$ areas lose lanthanum and form the RT5 reconstruction. A fully singly terminated $\mathrm{AlO}_{2}$ surface, on the other hand, is stable even at high temperatures.

\section{SUMMARY}

We investigated the surface of $\mathrm{LaAlO}_{3}[001]$ single crystals by low-energy electron microscopy and atomic force microscopy. Annealing from $930^{\circ} \mathrm{C}$ to $1150{ }^{\circ} \mathrm{C}$ in $\mathrm{UHV}$ as well as in air, we found a mixed ordered surface termination of unreconstructed $\mathrm{AlO}_{2}$ and reconstructed $(\sqrt{5} \times \sqrt{5} \mathrm{R} 26)$ $\mathrm{LaO}$, with the fraction of reconstructed $\mathrm{LaO}$ being about one-third. We could follow the formation of the mixed ordered termination with temperature from a mixture of small islands of either kind to stripes of $\mathrm{LaO}$ in the middle of terraces of $\mathrm{AlO}_{2}$, with the stripes lying lower than the surrounding terraces. By preparing a surface with a single $\mathrm{AlO}_{2}$ termination, we also demonstrated the difference in $I V$ characteristics for the unreconstructed $\mathrm{AlO}_{2}$ termination and the reconstructed $\mathrm{LaO}$ termination.

\section{ACKNOWLEDGMENTS}

We are grateful to Ruud Tromp for discussions and advice, and to Marcel Hesselberth and Daan Boltje for technical assistance. This work was supported by the Netherlands Organization for Scientific Research (NWO) by means of a "NWO Groot" grant. The work is part of the research program NWOnano, administered by the Foundation for Fundamental Research on Matter (FOM), which is part of NWO. 
[1] O. Ohtomo and H. Hwang, Nature (London) 427, 423 (2004).

[2] Dong-Wook Kim, Dae-Ho Kim, Bo-Soo Kang, T. W. Noh, D. R. Lee, and K.-B. Lee, Appl. Phys. Lett. 74, 2176 (1999).

[3] R. Gunnarsson, A. S. Kalabukhov, and D. Winkler, Surf. Sci. 603, 151 (2009).

[4] J. Yao, P. B. Merrill, S. S. Perry, D. Marton, and J. W. Rabalais, J. Chem. Phys. 108, 1645 (1998).

[5] P. A. W. van der Heide and J. W. Rabalais, Chem. Phys. Lett. 297, 350 (1998).

[6] R. J. Francis, S. C. Moss, and A. J. Jacobson, Phys. Rev. B 64, 235425 (2001).

[7] H. Kawanowa, H. Ozawa, M. Ohtsuki, Y. Gotoh, and R. Souda, Surf. Sci. 506, 87 (2002).

[8] D. A. Schmidt, Taisuke Ohta, Q. Yu, and M. A. Olmstead, J. Appl. Phys. 99, 113521 (2006).

[9] C. H. Lanier, J. M. Rondinelli, B. Deng, R. Kilaas, K. R. Poeppelmeier, and L. D. Marks, Phys. Rev. Lett. 98, 086102 (2007).
[10] R. M. Tromp, J. B. Hannon, A. W. Ellis, W. Wan, A. Berghaus, and O. Schaff, Ultramicroscopy 110, 852 (2010).

[11] S. M. Schramm, J. Kautz, A. Berghaus, O. Schaff, R. M. Tromp, and S. J. van der Molen, IBM J. Res. Dev. 55, 1 (2011).

[12] S. M. Schramm, A. B. Pang, M. S. Altman, and R. M. Tromp, Ultramicroscopy 115, 88 (2012).

[13] R. M. Tromp, J. B. Hannon, W. Wan, A. Berghaus, and O. Schaff, Ultramicroscopy 127, 25 (2013).

[14] J. B. Hannon, J. Sun, K. Pohl, and G. L. Kellogg, Phys. Rev. Lett. 96, 246103 (2006).

[15] A. K. Schmid, W. Schwieg, C. S. Rastomjee, B. Rausenberger, W. Engel, E. Zeitler, and A. M. Bradshaw, Surf. Sci. 331-333 Part A, 225 (1995).

[16] J. I. Flege and E. E. Krasovskii, Phys. Status Solidi 8, 463 (2014).

[17] T. Tachikawa, M. Minohara, Y. Nakanishi, Y. Hikita, M. Yoshita, H. Akiyama, C. Bell, and H. Y. Hwang, Appl. Phys. Lett. 101, 022104 (2012). 